\title{
Association between serum leptin levels and peritoneal dialysis: A meta-analysis
}

\author{
SHAN JIANG, KAI SONG, SHENG FENG and YONG-BIN SHI
}

Department of Nephrology, The Second Affiliated Hospital of Soochow University, Suzhou, Jiangsu 215000, P.R. China

Received June 18, 2014; Accepted February 13, 2015

DOI: 10.3892/etm.2015.2441

\begin{abstract}
There is limited information available with regard to the association between serum leptin levels, or other adipokines, and serum lipid levels and insulin sensitivity in patients undergoing peritoneal dialysis (PD). Thus, the aim of the present study was to perform a meta-analysis investigating this association. Potential relevant studies were identified through searching the following databases: MEDLINE, Science Citation Index, Cochrane Library, PubMed, Embase, CINAHL, Chinese Biomedical, Chinese Journal Full-Text and Weipu Journal. Statistical analyses were calculated using version 12.0 STATA software. In total, 21 case-control studies comprising 1,187 subjects (574 patients and 613 controls) were collected for the meta-analysis. The results identified a statistically significant difference in the serum levels of leptin when comparing the PD patients with the healthy controls [controls vs. cases, standardized mean difference (SMD), 2.09; 95\% confidence interval (CI), 1.58-2.59; $\mathrm{P}<0.001]$. Furthermore, ethnicity-subgroup analysis indicated that the PD patients of Asian and Caucasian populations were associated with increased serum levels of leptin (Asian population, SMD, 2.05; 95\% CI, 1.48-2.62; P<0.001; Caucasian population, SMD, 2.19; 95\% CI, 1.19-3.18; $\mathrm{P}<0.001)$. Therefore, serum leptin levels may be used as a prognostic marker for PD.
\end{abstract}

\section{Introduction}

Peritoneal dialysis (PD) is an effective and secure treatment method for patients with short-term (3-5 years) chronic kidney disease (CKD), and has been accepted as a form of renal replacement therapy (RRT) for end-stage renal disease (ESRD) patients (1). There are two forms of PD, consisting of automated peritoneal dialysis (APD) and continuous ambulatory peritoneal dialysis (CAPD). Certain studies have shown that APD has a lower mortality risk compared with CAPD,

Correspondence to: Dr Yong-Bin Shi, Department of Nephrology, The Second Affiliated Hospital of Soochow University, 1,055 Sanxiang Road, Suzhou, Jiangsu 215000, P.R. China

E-mail: su2h_syb@126.com

Key words: leptin, serum, peritoneal dialysis, meta-analysis with a mortality rate of $40 \%$ in patients undergoing APD and a rate of $60 \%$ in patients undergoing CAPD $(2,3)$. In 2008, an estimated 196,000 patients were undergoing PD worldwide, which represents $11 \%$ of the total dialysis population (4). In the United States, 368,000 patients underwent dialysis treatment in 2007 , with only a $7.2 \%$ PD prevalence (5). However, the number of patients undergoing treatment with $\mathrm{PD}$ appears to be increasing worldwide, with $41 \%$ of patients treated with PD in developed countries and 59\% of patients treated with PD in developing countries (4). PD, hemodialysis and kidney transplantation are the three major RRT modalities. In Hong Kong, PD is the first choice RRT for all ESRD patients (6,7). Due to the low cost, improved quality of life and excellent comparable survival rates, the use of PD has increased widely, and the treatment method has become an essential RRT for patients with ESRD (8). As an important risk factor contributing to diabetic foot ulceration, CKD, particularly with the enrollment of ESRD, has resulted in 200,000 patients receiving PD therapy worldwide, which is increasing by $>6 \%$ per annum $(9,10)$. These data indicate that the increasingly wide application of PD is playing a critical role in the treatment of ESRD patients (11). Furthermore, a previous study demonstrated that ESRD is associated with the endocrine function of adipose tissue, and high levels of leptin and adiponectin have been reported in patients with ESRD (12).

Leptin is a protein product of the obese gene and one of the adipocytokines primarily secreted by white adipocytes (13). The protein is formed of 167 amino acids and has a molecular weight of $16 \mathrm{kDa}(13)$. Leptin, via afferent signaling in the hypothalamus, is known to be involved in regulating the fat stored in the body and maintaining energy homeostasis, with the means of exerting influences on the sensation of hunger, energy intake and energy expenditure (14). Through increasing the utilization of glucose and the metabolism of oxidative glucose in adipocytes, insulin indirectly promotes leptin production (15). Serum concentrations of leptin are reported to be increased in obese individuals, due to a reduced ability to detect satiety, and have been associated with the fat content of the body (16). Serum leptin levels are also known to be higher in patients who are undergoing PD, such as patients with hemolytic uremic syndrome, when compared with individuals with normal renal function. This observation may be caused by the filtering of leptin at the glomerulus without obstruction and the degradation of the protein in the renal tubules, as a result of the impaired clearance by the kidney $(17,18)$. In 
addition, malnutrition is a dominant characteristic of uremic syndrome, and nutritional indicators, including the body mass index (BMI), the distribution of body fat and the plasma concentration of albumin, are known to be associated with the development of renal failure $(19,20)$. Inversely, decreased levels of total protein and albumin in renal failure patients may reflect the catabolism of protein, and hypoalbuminemia is strongly associated with malnutrition (21). Overexpression of serum leptin is hypothesized to be an independent risk factor for PD, due to the close association between peritonitis and the malnutrition resulting from chronic inflammation. PD may in certain cases be attributed to a higher expression of leptin clearance, as the plasma concentrations of leptin in the patients increase correspondingly (20). Previous studies have agreed with the hypothesis that hyperleptinemia is a leading cause of protein malnutrition, and that $\mathrm{PD}$ may be responsible for the higher levels of leptin $(22,23)$; however, alternative studies have reported that high serum concentrations of leptin may not be present in PD patients $(20,24)$. As a result, the aim of the present study was to perform a meta-analysis investigating the correlation between high plasma levels of leptin and PD.

\section{Materials and methods}

Search strategy. Potentially relevant studies were identified through a comprehensive literature search without language restriction, which covered the following computerized bibliographic databases: MEDLINE (1966-2014), Science Citation Index (1945-2014), Cochrane Library (Oxford, UK, Issue 12, 2014), PubMed (1966-2014), Embase (1974-2014) and CINAHL (1982-2014). In addition, the following three Chinese databases were included in the search to identify Chinese-language articles: Chinese Biomedical (1978-2014), Chinese Journal Full-Text (1980-2014) and Weipu Journal (1989-2014). The following medical subject headings and free language terms were used in conjunction with a highly sensitive search strategy. The search terms were as follows: 'PD' or 'Peritoneal Dialysis', 'Continuous Ambulatory PD' or 'CAPD', 'Continuous Cycling PD' or 'CCPD' or 'peritoneum dialysis', and 'Leptin', 'Obese Protein', 'Obese Gene Product', 'Ob Gene Product' or 'Ob Protein'. Additionally, reference lists of relevant studies selected from the electronic debates were searched manually to identify additional studies.

Inclusion and exclusion criteria. To be included in the systematic review, retrieved studies were assessed for their suitability in meeting the following criteria: i) Search results were conducted within a human population and published in a peer-reviewed journal; ii) only case-control studies examining the association between serum leptin levels and patients undergoing PD were incorporated into the meta-analysis; iii) all the patients satisfied the guideline criteria for PD (25); iv) articles were required to present original data and supply sufficient information with regard to the serum leptin levels; and v) when studies provided overlapping data, the study that had the largest sample number was selected. The major exclusion criteria in this systematic review were as follows: i) Articles that did not satisfy the current inclusion criteria; ii) certain publication types, including letters, abstracts, reviews, meta-analyses and proceedings; iii) unpublished sources of data; iv) duplication publications or studies without extractable, numerical data; and v) subgroup analysis of the included trials. With the application of these inclusion criteria, the title and abstract of all the articles were evaluated on relevance. From the selected articles, the full texts were reviewed, followed by a decision on their eligibility for inclusion.

Study quality and data extraction. In order to ensure consistency in reviewing and reporting the results, two reviewers independently assessed the methodological quality of the included trials using the Newcastle-Ottawa Scale (NOS) criteria with regard to study design, content and ease-of-use in the explanation of results or the meta-analysis for assessing the quality (26). Three broad perspectives were judged, including subject selection (0-4), subject comparability (0-2) and clinical outcome (0-3). The subject selection criteria included four sub-criteria: i) Adequacy of case definition; ii) representativeness of the cases; iii) selection of control; and iv) definition of controls. Subject comparability comprised a single critera; the comparability of cases and controls on the basis of the design or analysis. Clinical outcome consisted of three sub-criteria; the ascertainment of clinical outcome, a consistent method of ascertainment for cases and controls and non-response rate (http://www.biomedcentral.com/1471-2288/14/45). The NOS scores ranged between 0 and 9; a study was classified as good quality for the evidence with a score of $\geq 7$.

Each of the two reviewers assessed the studies independently based on the aforementioned inclusion/exclusion criteria. A standardized data form in duplicate was used to collect the following descriptive information of the included studies: Surname and initials of the first author, the year of publication or submission, journal, source country, racial descent of the study population, language of publication, study design, number of cases and controls, demographic variables of the subjects, detection method of the serum leptin levels and baseline leptin levels in the cases and the controls. Disagreement on the inclusion of a single study was settled by discussion, or a third investigator was consulted.

Statistical analysis. The effect size was represented by the mean \pm standard difference, which was used to calculate the serum leptin levels in patients undergoing PD and healthy controls. A confidential interval of $95 \%$ (95\% CI) was calculated for all mean values, using the Z-test. In addition, a test for the heterogeneity between the included trials for each comparison was performed using the Cochran's Q test and $\mathrm{I}^{2}$ tests (27). If the $\mathrm{Q}$ test showed evidence of a P-value of $<0.05$ or if the $\mathrm{I}^{2}$ test exhibited a value of $>50 \%$, which indicated maximal heterogeneity among the included studies, a meta-regression analysis with a random-effects model was conducted to investigate the sources of heterogeneity, while in other cases, the SMD values were pooled in accordance with the fixed-effects model $(28,29)$. When substantial heterogeneity was identified, the differences in the leptin levels (and 95\% CI) were evaluated among subgroups for different explanatory variables. Additionally, in order to evaluate the impact of single studies on the overall estimate, a one-way sensitivity analysis was employed. Furthermore, Egger's linear regression test, with visual inspection of the funnel plot, was applied to detect the potential publication bias $(30,31)$. 


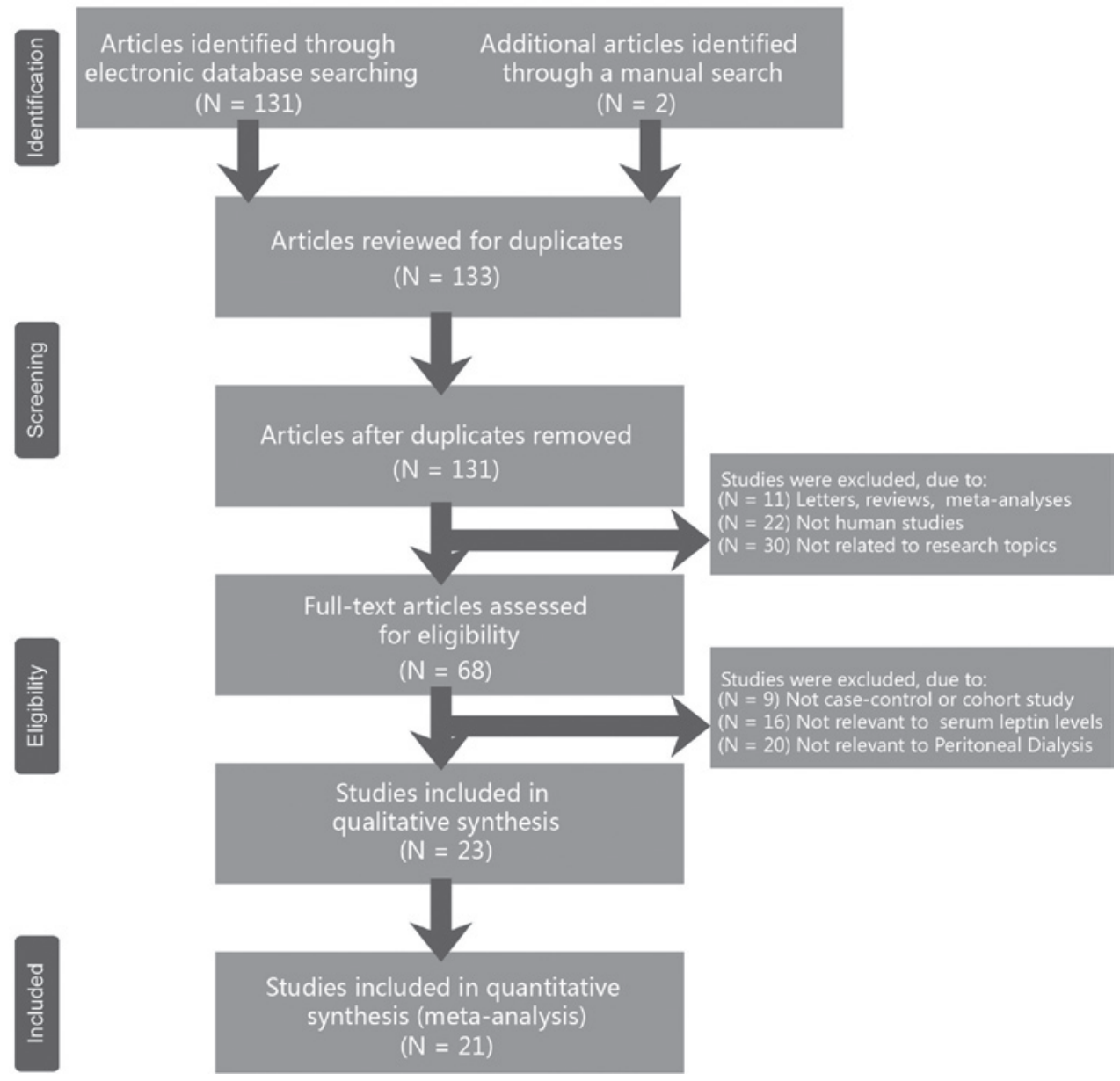

Figure 1. Flow chart showing the literature search and study selection procedure. In total, 21 case-control studies were included in the meta-analysis.

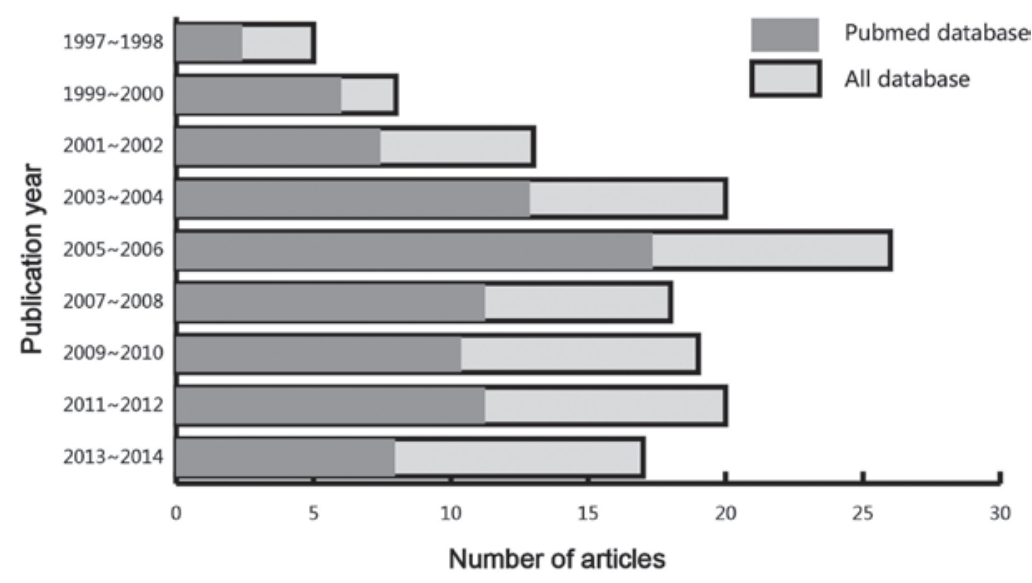

Figure 2. Distribution of topic-related literature in an electronic database over the last decade.

Statistical analyses were conducted using STATA statistical software (version 12.0; Stata Corporation, College Station, TX, USA).

\section{Results}

Description of the included studies. The combined electronic and manual search initially resulted in 133 potentially eligible articles. Following the identification of two duplicated studies, the retrieved studies $(n=131)$ were screened by their title and abstract for relevance. Subsequently, 63 irrelevant articles were excluded. The remaining 68 articles that qualified for full-text reading were systematically reviewed. After reading the full text, 45 articles were deemed unsuitable and were therefore excluded. Thus, 23 articles were included in the qualitative analysis. However, an additional two studies were excluded due to lack of data integrity following a more careful assessment of the remaining articles. A flow diagram of the study selection progress and the main reasons for exclusion is shown in Fig. 1 Finally, 21 case-control studies, comprising 
Table I. Characteristics of the included studies focused on the serum levels of leptin.

\begin{tabular}{|c|c|c|c|c|c|c|c|c|c|c|}
\hline \multirow{2}{*}{$\begin{array}{l}\text { First author } \\
\text { (reference) }\end{array}$} & \multirow[b]{2}{*}{ Year } & \multirow[b]{2}{*}{ Ethnicity } & \multicolumn{2}{|c|}{ Sample size (n) } & \multicolumn{2}{|c|}{ Gender, M/F (n) } & \multicolumn{2}{|c|}{ Mean age (years) } & \multirow[b]{2}{*}{ Method } & \multirow{2}{*}{$\begin{array}{l}\text { NOS } \\
\text { score }\end{array}$} \\
\hline & & & Case & Control & Case & Control & Case & Control & & \\
\hline Kaynar K (20) & 2014 & Asian & 30 & 30 & - & - & $39.1 \pm 13.4$ & $33.4 \pm 9.4$ & ELISA & \\
\hline Yang FF (40) & 2011 & Asian & 30 & 30 & $17 / 13$ & - & $61.7 \pm 14.0$ & - & RIA & \\
\hline Wu R (42) & 2011 & Asian & 26 & 30 & $15 / 11$ & - & $53.3 \pm 12.7$ & - & ELISA & \\
\hline Ma Y (46) & 2011 & Asian & 20 & 20 & $12 / 8$ & - & $55.8 \pm 14.5$ & - & RIA & \\
\hline Zhang L (24) & 2010 & Asian & 20 & 13 & $11 / 9$ & $6 / 7$ & $58.8 \pm 11.4$ & $57.8 \pm 12.7$ & RIA & \\
\hline Wang ZM (44) & 2010 & Asian & 45 & 30 & $24 / 21$ & $17 / 13$ & $60.4 \pm 10.2$ & $58.5 \pm 12.9$ & RIA & \\
\hline Malyszko J (35) & 2010 & Caucasian & 40 & 22 & - & $11 / 9$ & $52.3 \pm 12.5$ & $56.0 \pm 14.8$ & RIA & \\
\hline Małgorzewicz S (36) & 2010 & Caucasian & 30 & 23 & $12 / 18$ & $8 / 15$ & $57.3 \pm 16.6$ & $63.3 \pm 7.8$ & ELISA & \\
\hline Xu XD (41) & 2008 & Asian & 40 & 40 & - & - & - & - & RIA & \\
\hline Taskapan MC (15) & 2007 & Asian & 30 & 30 & $18 / 12$ & $17 / 13$ & $43.2 \pm 10.8$ & $40.9 \pm 10.0$ & ELISA & \\
\hline Wu JQ (43) & 2006 & Asian & 26 & 26 & - & - & - & - & ELISA & \\
\hline Guan X (47) & 2006 & Asian & 30 & 30 & $16 / 14$ & $19 / 11$ & $52.3 \pm 3.1$ & $51.2 \pm 3.6$ & ELISA & \\
\hline Buyan N (39) & 2006 & Asian & 24 & 23 & - & - & $13(7-18)$ & $14(4-18)$ & RIA & \\
\hline Wright M (32) & 2004 & Caucasian & 39 & 43 & $23 / 16$ & $22 / 21$ & $53(23-75)$ & $50(36-73)$ & ELISA & . \\
\hline Hilkens MG (22) & 2003 & Caucasian & 10 & 10 & $5 / 5$ & $5 / 5$ & 49 & 49 & ELISA & \\
\hline Zuo J (45) & 2002 & Asian & 9 & 13 & $8 / 1$ & $8 / 5$ & $61.0 \pm 12.0$ & $52.0 \pm 6.0$ & ELISA & \\
\hline Vignioble M (33) & 2001 & Caucasian & 23 & 35 & $10 / 13$ & $16 / 19$ & - & - & RIA & \\
\hline Tsujimoto Y (34) & 1999 & Asian & 46 & 67 & $28 / 18$ & $37 / 30$ & $50.0 \pm 1.6$ & $50.6 \pm 1.0$ & ELISA & 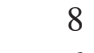 \\
\hline Landt M (37) & 1999 & Caucasian & 28 & 28 & $14 / 14$ & $14 / 14$ & $54.5 \pm 17.7$ & $36.8 \pm 8.0$ & RIA & \\
\hline Johansen KL (38) & 1998 & Caucasian & 9 & 41 & $5 / 4$ & $21 / 20$ & $43.0 \pm 15.0$ & $36.0 \pm 10.0$ & RIA & \\
\hline Howard JK (23) & 1997 & Caucasian & 19 & 29 & - & - & - & - & RIA & \\
\hline
\end{tabular}

M, male; F, female; ELISA, enzyme-linked immunosorbent assay; RIA, radioimmunoassay; NOS, Newcastle-Ottawa Scale.

574 patients that had undergone PD and 613 controls, were incorporated into the current meta-analysis $(15,20,22-24,3$ 2-47). All the eligible studies had been published between 1997 and 2014 (Fig. 2), and all the enrolled papers exhibited moderate to high quality.

With regard to the demographic variables of the 21 included studies, 13 studies were performed with an Asian study population, while the remaining eight studies included a Caucasian study population. With regard to the methods used to detect the leptin levels, 11 studies utilized a radioimmunoassay (RIA), while the remaining 10 studies performed an enzyme-linked immunosorbent assay (ELISA). Table I presents the baseline characteristics of the study populations and the characteristics of the included studies.

Quantitative data synthesis. In the meta-analysis, the serum leptin levels in the patients receiving PD and the controls were analyzed using a random effects model due to the evidence of heterogeneity (controls vs. cases, $\mathrm{I}^{2}=91.8 \%, \mathrm{P}<0.001$ ). Additionally, since a significant heterogeneity was shown to exist, the studies were stratified by ethnicity (Asian population subgroup, $\mathrm{I}^{2}=89.9 \%, \mathrm{P}<0.001$; Caucasian population subgroup, $\left.\mathrm{I}^{2}=93.9 \%, \mathrm{P}<0.001\right)$ and detection methods (ELISA subgroup, $\mathrm{I}^{2}=90.9 \%, \mathrm{P}<0.001$; RIA subgroup, $\left.\mathrm{I}^{2}=92.3 \%, \mathrm{P}<0.001\right)$. Results from the meta-analysis revealed a statistically significant difference in the serum levels of leptin between the PD patients and the healthy controls. Higher serum concentrations of leptin were observed in the PD patients when compared with the controls, according to the random effects pooled SMD in the 21 included studies (controls vs. cases, SMD, 2.09; 95\% CI, 1.58-2.59; P<0.001; Fig. 3).

With regard to the ethnicity-stratified subgroup analysis, the results from the meta-analysis revealed that the PD patients were associated with increased serum leptin levels in contrast to the healthy controls in the Asian population subgroup (controls vs. cases, SMD, 2.05; 95\% CI, 1.48-2.62; $\mathrm{P}<0.001$ ). Additionally, higher serum leptin levels were observed more frequently in the PD patients in the Caucasian population subgroup (controls vs. cases, SMD, 2.19; 95\% CI, 1.19-3.18; $\mathrm{P}<0.001$ ). Furthermore, in the method-stratified subgroup analysis, the PD patients were found to have higher serum leptin levels in the ELISA subgroup (controls vs. cases, SMD, 1.86; 95\% CI, 1.19-2.52; $\mathrm{P}<0.001$ ), and a similar correlation was also observed in the RIA subgroup (controls vs. cases, SMD, 2.29; 95\% CI, 1.53-3.06; P<0.001; Fig. 4).

Further sensitivity analyses were conducted to determine whether the review conclusions were affected by the selection of a single study, and the findings indicated that no single study had an effect on the pooled SMD values in the current meta-analysis (Fig. 5). Finally, Egger's regression analysis displays the asymmetrical distribution of the funnel plot, which indicates publication biases in the differences in serum leptin levels between patients undergoing PD and controls $(\mathrm{t}=4.87 ; \mathrm{P}<0.001)$ in the systematic reviews (Fig. 6). 


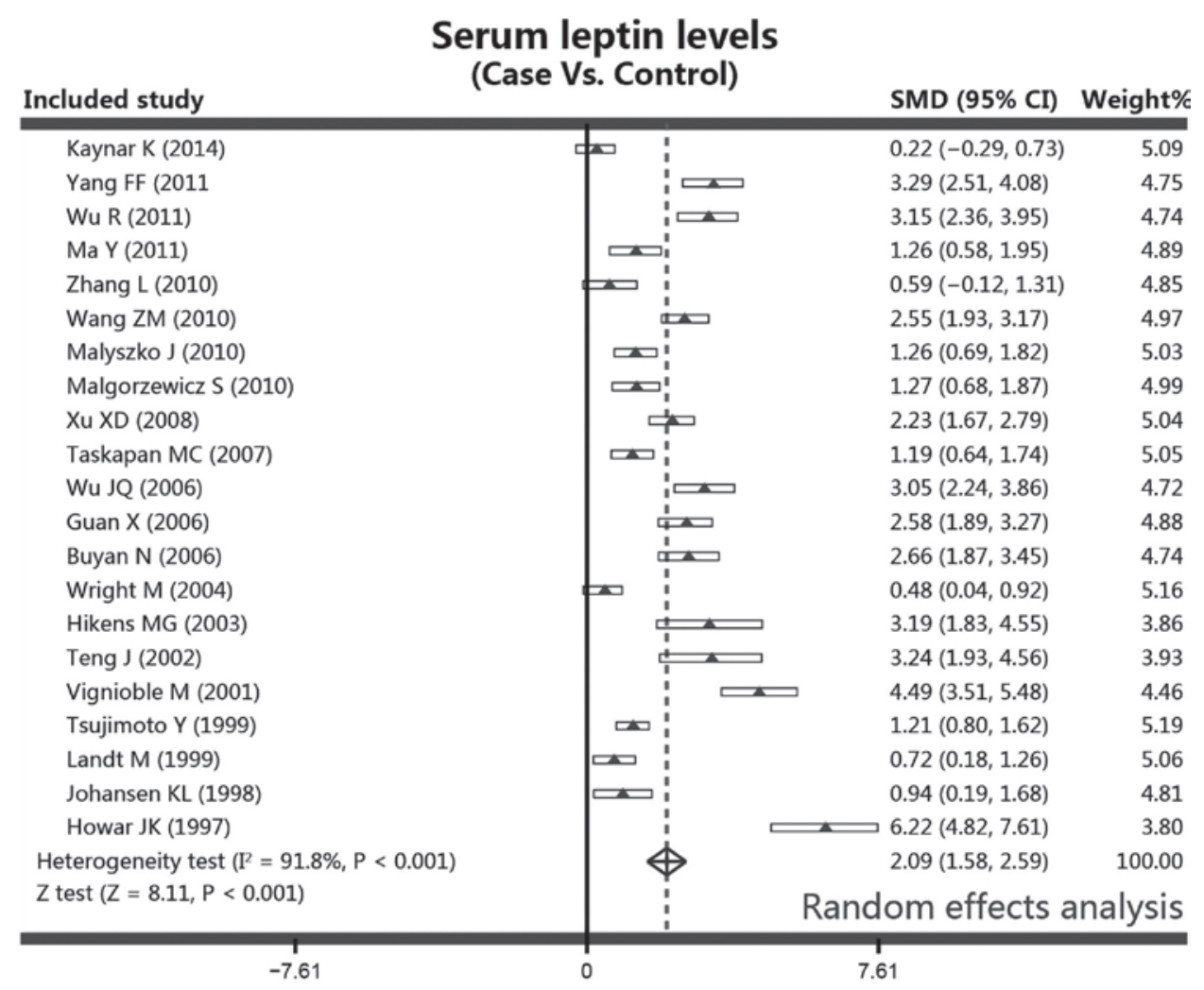

Figure 3. Forest plot showing the differences in the serum leptin levels between patients undergoing peritoneal dialysis and healthy controls. CI, confidence interval; SMD, standardized mean difference.

\section{Discussion}

The aim of the present meta-analysis was to investigate the association between high serum levels of leptin and PD. The results of the meta-analysis demonstrated that high leptin levels were closely associated with PD, indicating that PD therapy may not be an effective therapy for the clearance of leptin. As the first adipocyte-derived hormone, leptin is a $16 \mathrm{kDa}$ peptide hormone with 146 amino acids, which is formed by cleaving a signal peptide of 21 amino acids from its prototype in the blood (48). Leptin plays a role in the central nervous system through hypothalamic pathways, with its main function to cause a decrease in food intake and an increase in the metabolic rate, promoting weight loss and the regulation of the energy balance (39). In addition to the effect on the central nervous system, leptin is also able to inhibit the secretion of insulin, increase natriuresis, diuresis and angiogenesis, and promote the calcification of vessels and increase oxidative stress (49). Thus, leptin is associated with a number of diseases, such as coronary artery calcification, vascular dysfunction, hypertension and kidney diseases (50). The metabolic pathway of leptin is through glomerulus filtration, followed by degradation within the renal tubules; thus, a high serum level of leptin is often observed in kidney disease patients (51). Elevated serum leptin levels may cause weight loss, malnutrition and anorexia, which can deteriorate the symptoms of kidney disease patients and are detrimental for the long-term survival of patients $(36,52)$. PD, as a first-choice RRT, uses the peritoneum as a dialysis membrane, and is a safe and gentle method to correct metabolic and electrolytic disturbances generated in kidney diseases, including the clearance of leptin (53). However, PD is only partially able to clear leptin, and the clearance volume in PD and the high level of leptin have been found to be positively associated (54). In PD patients, the filtration rate of the glomerulus is decreased due to renal impairment; thus, the renal clearance of leptin is decreased, which leads to high serum leptin levels (55). Furthermore, the increased glucose load results in chronic hyperinsulinemia, which subsequently stimulates the insulin level and regulates the gene expression of leptin, ultimately causing high leptin serum levels (15). From these observations, it was hypothesized that high leptin levels in kidney disease patients may deteriorate the symptoms of the patients by causing malnutrition and anorexia. In addition, PD was hypothesized to be closely associated with the high leptin level, through the insufficient physical clearance, renal impairment and the increase in the indirect glucose load, which subsequently regulated the increased expression of leptin. Malyszko et al also observed increased levels of leptin in PD patients, which represented a connection between inflammation and adipocytokines, and the authors concluded that the dialysis time and adequacy may affect the clearance of leptin in patients undergoing dialysis (35).

Since a number of factors may influence the association between high serum levels of leptin and PD, a stratified analysis based on the ethnicity of the study population and the leptin detection method was established. The ethnicity subgroup analysis revealed a significant association between the leptin serum level increase and PD therapy in Asian and Caucasian populations, which may demonstrate that no racial difference exists between the high leptin level 
Serum leptin levels (Ethnicity: Case VS. Control)

\begin{tabular}{|c|c|c|c|}
\hline \multicolumn{2}{|c|}{ Included study } & SMD (95\% Cl) & \multirow[t]{2}{*}{ Weight $\%$} \\
\hline \multicolumn{3}{|l|}{ Asians } & \\
\hline Kaynar K (2014) & - & $0.22(-0.29,0.73)$ & 5.09 \\
\hline Yang FF (2011) & صـ & $3.29(2.51,4.08)$ & 4.75 \\
\hline Wu R (2011) & صـ & $3.15(2.36,3.95)$ & 4.74 \\
\hline Ma Y (2011) & إ' & $1.26(0.58,1.95)$ & 4.89 \\
\hline Zhang L (2010) & i & $0.59(-0.12,1.31)$ & 4.85 \\
\hline Wang ZM (2010) & & $2.55(1.93,3.17)$ & 4.97 \\
\hline Xu XD (2008) & 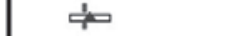 & $2.23(1.67,2.79)$ & 5.04 \\
\hline Taskapan MC (2007) & $\infty$ & $1.19(0.64,1.74)$ & 5.05 \\
\hline Wu JQ (2006) & صـ & $3.05(2.24,3.86)$ & 4.72 \\
\hline Guan X (2006) & & $2.58(1.89,3.27)$ & 4.88 \\
\hline Buyan N (2006) & صه & $2.66(1.87,3.45)$ & 4.74 \\
\hline Teng J (2002) & & $3.24(1.93,4.56)$ & 3.93 \\
\hline Tsujimoto Y (1999) & $\infty$ & $1.21(0.80,1.62)$ & 5.19 \\
\hline $\begin{array}{l}\text { Heterogeneity test }\left(I^{2}=89.9 \%, P<0.001\right) \\
Z \text { test }(Z=7.04, P<0.001)\end{array}$ & & $2.05(1.48,2.62)$ & 62.83 \\
\hline \multicolumn{4}{|l|}{ Caucasians } \\
\hline Malyszko J (2010) & '; & $1.26(0.69,1.82)$ & 5.03 \\
\hline Malgorzewicz S (2010) & $\infty$ & $1.27(0.68,1.87)$ & 4.99 \\
\hline Wright M (2004) & : i & $0.48(0.04,0.92)$ & 5.16 \\
\hline Hikens MG (2003) & $=$ & $3.19(1.83,4.55)$ & 3.86 \\
\hline Vignioble M (2001) & $\square$ & $4.49(3.51,5.48)$ & 4.46 \\
\hline Landt M (1999) & $\infty$ & $0.72(0.18,1.26)$ & 5.06 \\
\hline Johansen KL (1998) & ص & $0.94(0.19,1.68)$ & 4.81 \\
\hline Howar JK (1997) & $\Longrightarrow$ & D $6.22(4.82,7.61)$ & 3.80 \\
\hline $\begin{array}{l}\text { Heterogeneity test }\left(\mathbb{R}^{2}=93.9 \%, P<0.001\right) \\
Z \text { test }(\mathrm{Z}=4.32, \mathrm{P}<0.001)\end{array}$ & & $2.19(1.19,3.18)$ & 37.17 \\
\hline Heterogeneity test $\left(\mathbb{I}^{2}=91.8 \%, \mathrm{P}<0.001\right)$ & & $2.09(1.58,2.59)$ & 100.00 \\
\hline$Z$ test $(Z=8.11, P<0.001)$ & \multicolumn{3}{|c|}{ Random effects analysis } \\
\hline-7.61 & 0 & 7.61 & \\
\hline \multicolumn{4}{|c|}{$\begin{array}{l}\text { Serum leptin levels } \\
\text { (Method: Case VS. Control) }\end{array}$} \\
\hline \multicolumn{4}{|l|}{ ELSA } \\
\hline Kaynar K (2014) & ص & $0.22(-0.29,0.73)$ & 5.09 \\
\hline Wu R (2011) & כ & $3.15(2.36,3.95)$ & 4.74 \\
\hline Malgorzewicz S (2010) & a & $1.27(0.68,1.87)$ & 4.99 \\
\hline Taskapan MC (2007) & $\infty$ & $1.19(0.64,1.74)$ & 5.05 \\
\hline Wu JQ (2006) & ص & $3.05(2.24,3.86)$ & 4.72 \\
\hline Guan X (2006) & & $2.58(1.89,3.27)$ & 4.88 \\
\hline Wright M (2004) & ot & $0.48(0.04,0.92)$ & 5.16 \\
\hline Hikens MG (2003) & $=$ & $3.19(1.83,4.55)$ & 3.86 \\
\hline Teng J (2002) & & $3.24(1.93,4.56)$ & 3.93 \\
\hline Tsujimoto Y (1999) & $\infty$ & $1.21(0.80,1.62)$ & 5.19 \\
\hline $\begin{array}{l}\text { Heterogeneity test }\left(I^{2}=90.9 \%, P<0.001\right) \\
Z \text { test }(Z=5.49, P<0.001)\end{array}$ & & $1.86(1.19,2.52)$ & 47.61 \\
\hline \multicolumn{4}{|l|}{ RIA } \\
\hline Yang FF (2011) & صـ & $3.29(2.51,4.08)$ & 4.75 \\
\hline $\operatorname{MaY}(2011)$ & $\infty$; & $1.26(0.58,1.95)$ & 4.89 \\
\hline Zhang L (2010) & ; & $0.59(-0.12,1.31)$ & 4.85 \\
\hline Wang ZM (2010) & & $2.55(1.93,3.17)$ & 4.97 \\
\hline Malyszko J (2010) & $\infty$; & $1.26(0.69,1.82)$ & 5.03 \\
\hline Xu XD (2008) & هم & $2.23(1.67,2.79)$ & 5.04 \\
\hline Buyan N (2006) & صـ & $2.66(1.87,3.45)$ & 4.74 \\
\hline Vignioble M (2001) & 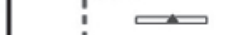 & $4.49(3.51,5.48)$ & 4.46 \\
\hline Landt M (1999) & $\Leftrightarrow$ ! & $0.72(0.18,1.26)$ & 5.06 \\
\hline Johansen KL (1998) & ; & $0.94(0.19,1.68)$ & 4.81 \\
\hline Howar JK (1997) & 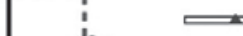 & $6.22(4.82,7.61)$ & 3.80 \\
\hline $\begin{array}{l}\text { Heterogeneity test }(R=92.3 \%, P<0.001) \\
Z \text { test }(Z=5.86, P<0.001)\end{array}$ & & $2.29(1.53,3.06)$ & 52.39 \\
\hline Heterogeneity test $(R=91.8 \%, P<0.001)$ & $\oplus$ & $2.09(1.58,2.59)$ & 100.00 \\
\hline$Z$ test $(Z=8.11, P<0.001)$ & \multicolumn{3}{|c|}{ Random effects analysis } \\
\hline
\end{tabular}

Figure 4. Subgroup analyses indicating the differences in the serum leptin levels between patients undergoing peritoneal dialysis and healthy controls. CI, confidence interval; SMD, standardized mean difference; ELISA, enzyme-linked immunosorbent assay; RIA, radioimmunoassay.

and PD. Therefore, the results of the present study are in accordance with previous studies that demonstrated a close connection between PD therapy and an increase in serum leptin levels $(19,22,43)$. These observations indicate that PD therapy may contribute potential damage to renal function and subsequently have an influence on the clearance of leptin; thus, additional measures should be undertaken to reduce the leptin level in PD patients and improve the treatment of kidney disease.

However, there were a number of limitations in the current meta-analysis that should be taken into consideration. Firstly, the existence of heterogeneity, since the groups were not homogenous with respect to age, BMI, smoking status, ethnicity and serum level detection methods, and the insulin sensitivity 


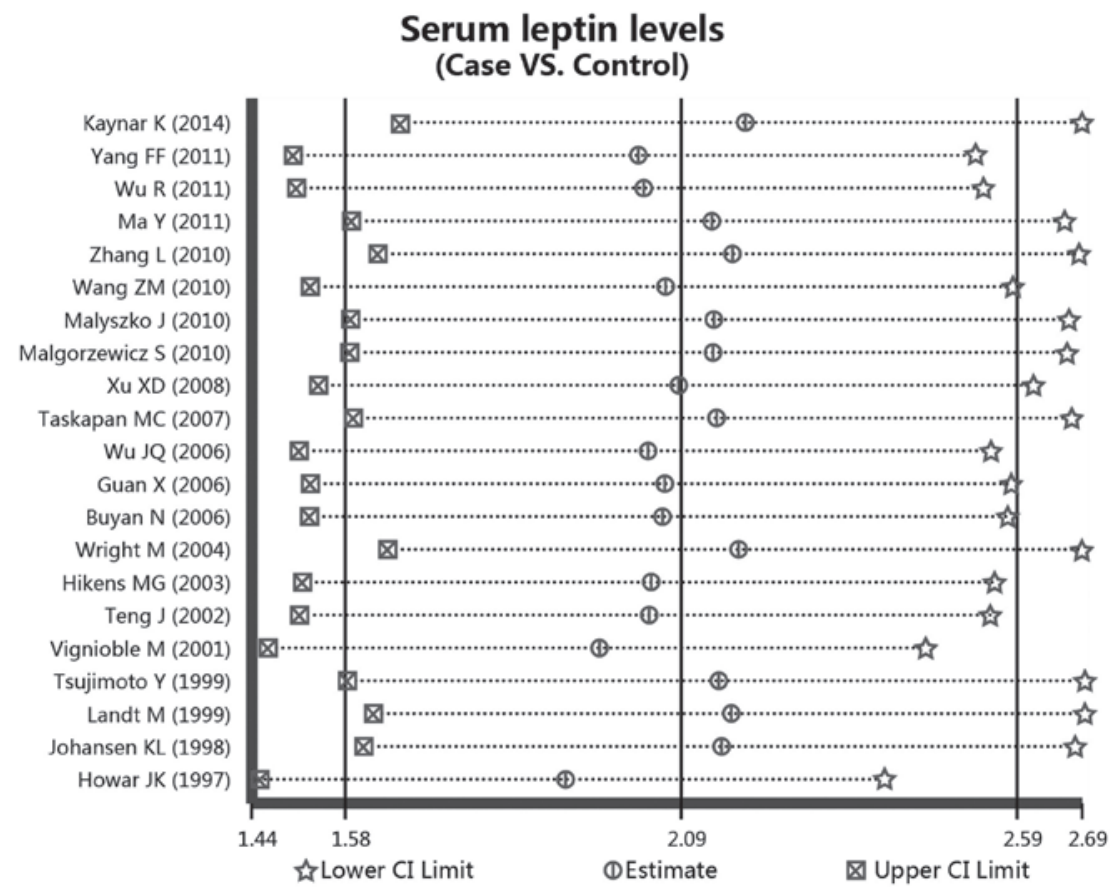

Figure 5. Sensitivity analysis of the standardized mean difference coefficients of the differences in the serum leptin levels between patients undergoing peritoneal dialysis and healthy controls. CI, confidence interval.

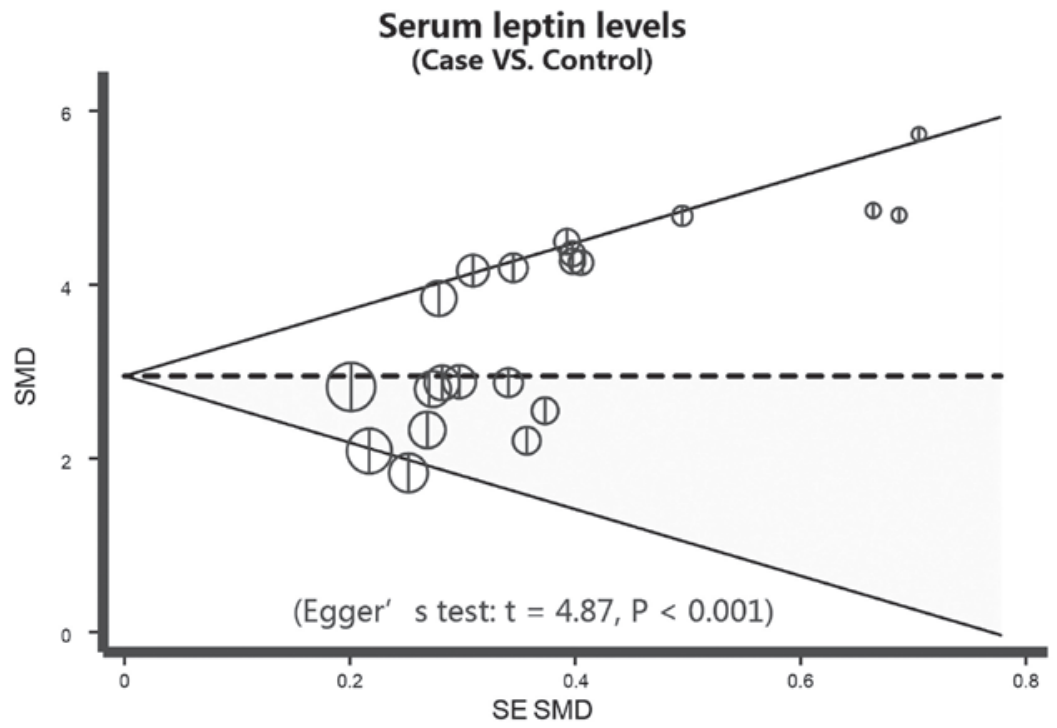

Figure 6. Funnel plot showing the publication biases with regard to the differences in the serum leptin levels between patients undergoing peritoneal dialysis and healthy controls. SMD, standardized mean difference; SE, standard error.

of the patients was not further investigated. Notably, certain publication convention may be acknowledged that positive results tend to be more acceptable by journals, while negative researches are often easy to be rejected or not submitted for review. Thus, the lack of negative results may restrict a broader experiment and constrict the findings of the present study to a large extent. Furthermore, language introduces bias and those publications prone to be published in English language-based journals. Thirdly, although this study was based on relatively large sample size studies, a relatively small sample size may limit the detection of more subtle changes over time. In addition, regardless of the underlying degree of glucose tolerance, fasting or diet, significant circadian fluctuations in the serum leptin concentration may obscure a number of small, but significant changes, in the serum leptin level, which may be a source of potential bias for longitudinal observation. Therefore, all these factors may result in an inconsistent outcome.

In conclusion, the present study reported increased serum leptin concentrations in patients undergoing PD therapy, indicating that PD therapy may contribute potential damage to renal function and affect the clearance of leptin. These results highlight the importance of leptin as a potential determinant of weight loss, malnutrition and anorexia, particularly in patients undergoing PD therapy. However, further studies with 
larger sample sizes are required to confirm the clinical utility of serum leptin as an important biomarker for PD patients.

\section{References}

1. Chaudhary $\mathrm{K}$ and Khanna R: Biocompatible peritoneal dialysis solutions: do we have one? Clin J Am Soc Nephrol 5: 723-732, 2010.

2. Katavetin P, Theerasin Y, Treamtrakanpon W, et al: Treatment failure in automated peritoneal dialysis and double-bag continuous ambulatory peritoneal dialysis. Nephrology (Carlton) 18: 545-548, 2013.

3. Cortés-SanabriaL,Paredes-CeseñaCA,Herrera-Llamas RM, etal: Comparison of cost-utility between automated peritoneal dialysis and continuous ambulatory peritoneal dialysis. Arch Med Res 44: 655-661, 2013.

4. Jain AK, Blake P, Cordy P and Garg AX: Global trends in rates of peritoneal dialysis. J Am Soc Nephrol 23: 533-544, 2012.

5. Chaudhary K, Sangha H and Khanna R: Peritoneal dialysis first: rationale. Clin J Am Soc Nephrol 6: 447-456, 2011.

6. Sakthong P and Kasemsup V: Health utility measured with EQ-5D in Thai patients undergoing peritoneal dialysis. Value Health 15 (Suppl): S79-S84, 2012.

7. Kwan BC, Chow KM, Ma TK, et al: Automated peritoneal dialysis in Hong Kong: there are two distinct groups of patients. Nephrology (Carlton) 18: 356-364, 2013.

8. Guney I, Solak Y, Atalay H, et al: Comparison of effects of automated peritoneal dialysis and continuous ambulatory peritoneal dialysis on health-related quality of life, sleep quality and depression. Hemodial Int 14: 515-522, 2010.

9. Johnson DW, Brown FG, Clarke M, et al; balANZ Trial Investigators: Effects of biocompatible versus standard fluid on peritoneal dialysis outcomes. J Am Soc Nephrol 23: 1097-1107, 2012.

10. Kaminski M, Frescos N and Tucker S: Prevalence of risk factors for foot ulceration in patients with end-stage renal disease on haemodialysis. Intern Med J 42: e120-e128, 2012.

11. Weinhandl ED, Foley RN, Gilbertson DT, et al: Propensity-matched mortality comparison of incident hemodialysis and peritoneal dialysis patients. J Am Soc Nephrol 21: 499-506, 2010

12. Kim S, Oh KH, Chin HJ, et al: Effective removal of leptin via hemodiafiltration with on-line endogenous reinfusion therapy. Clin Nephrol 72: 442-448, 2009.

13. Marwarha G and Ghribi O: Leptin signaling and Alzheimer's disease. Am J Neurodegener Dis 1: 245-265, 2012.

14. Mutabaruka MS, Aoulad Aissa M, Delalandre A, et al: Local leptin production in osteoarthritis subchondral osteoblasts may be responsible for their abnormal phenotypic expression. Arthritis Res Ther 12: R20, 2010.

15. Taskapan MC, Taskapan H, Sahin I, et al: Serum leptin, resistin and lipid levels in patients with end stage renal failure with regard to dialysis modality. Ren Fail 29: 147-154, 2007.

16. Fang TC, Lee CJ, Wang $\mathrm{CH}$, et al: Fasting serum leptin level correlates with mid-arm fat area in peritoneal dialysis patients. Ther Apher Dial 14: 583-588, 2010.

17. Briley LP and Szczech LA: Leptin and renal disease. Semin Dial 19: 54-59, 2006.

18. Mak RH, Cheung W, Cone RD and Marks DL: Leptin and inflammation-associated cachexia in chronic kidney disease. Kidney Int 69: 794-797, 2006.

19. Pérez-Fontán M, Cordido F, Rodríguez-Carmona A, et al: Plasma ghrelin levels in patients undergoing haemodialysis and peritoneal dialysis. Nephrol Dial Transplant 19: 2095-2100, 2004.

20. Kaynar K, Kural BV, Ulusoy S, et al: Is there any interaction of resistin and adiponectin levels with protein-energy wasting among patients with chronic kidney disease. Hemodial Int 18 $153-162,2014$

21. Horwich TB, Kalantar-Zadeh K, MacLellan RW and Fonarow GC: Albumin levels predict survival in patients with systolic heart failure. Am Heart J 155: 883-889, 2008.

22. Hilkens MG, Netea MG, Van der Meer JW and Koolen MI: Leptin and proinflammatory cytokines in patients undergoing peritoneal dialysis. Eur J Clin Invest 33: 525-526, author reply 527-528, 2003.

23. Howard JK, Lord GM, Clutterbuck EJ, et al: Plasma immunoreactive leptin concentration in end-stage renal disease. Clin Sci (Lond) 93: 119-126, 1997.
24. Zhang L, Liu J, Xu DM, et al: Clearance effect of peritoneal dialysis on leptin. J Southeast Univ 29: 539-542, 2010.

25. Tsubakihara Y, Nishi S, Akiba T, et al: 2008 Japanese society for dialysis therapy: guidelines for renal anemia in chronic kidney disease. Ther Apher Dial 14: 240-275, 2010.

26. Stang A: Critical evaluation of the Newcastle-Ottawa scale for the assessment of the quality of nonrandomized studies in meta-analyses. Eur J Epidemiol 25: 603-605, 2010.

27. Zintzaras E and Ioannidis JP: HEGESMA: genome search meta-analysis and heterogeneity testing. Bioinformatics 21 : 3672-3673, 2005

28. Zintzaras E and Ioannidis JP: Heterogeneity testing in meta-analysis of genome searches. Genet Epidemiol 28: 123-137, 2005.

29. Higgins JP and Thompson SG: Quantifying heterogeneity in a meta-analysis. Stat Med 21: 1539-1558, 2002

30. Song F and Gilbody S: Bias in meta-analysis detected by a simple, graphical test. Increase in studies of publication bias coincided with increasing use of meta-analysis. BMJ 316: 471, 1998.

31. Peters JL, Sutton AJ, Jones DR, et al: Comparison of two methods to detect publication bias in meta-analysis. JAMA 295: 676-680, 2006.

32. Wright M, Woodrow G, O'Brien S, et al: Cholecystokinin and leptin: their influence upon the eating behaviour and nutrient intake of dialysis patients. Nephrol Dial Transplant 19: 133-140, 2004.

33. Vignioble M, Brichard S, Jadoul M and Goffin E: Serum leptin concentration in peritoneal dialysis patients: determinants, longitudinal evolution and circadian rhythm. Acta Clin Belg 56: 173-179, 2001.

34. Tsujimoto Y, Shoji T, Tabata T, et al: Leptin in peritoneal dialysate from continuous ambulatory peritoneal dialysis patients. Am J Kidney Dis 34: 832-838, 1999.

35. Malyszko J, Malyszko JS and Mysliwiec M: Visfatin and endothelial function in dialyzed patients. Nephrology (Carlton) 15: 190-196, 2010.

36. Małgorzewicz S, Lichodziejewska-Niemierko M, Aleksandrowicz-Wrona E, et al: Adipokines, endothelial dysfunction and nutritional status in peritoneal dialysis patients. Scand J Urol Nephrol 44: 445-451, 2010.

37. Landt M, Parvin CA, Dagogo-Jack S, et al: Leptin elimination in hyperleptinaemic peritoneal dialysis patients. Nephrol Dial Transplant 14: 732-737, 1999.

38. Johansen KL, Mulligan K, Tai V and Schambelan M: Leptin, body composition and indices of malnutrition in patients on dialysis. J Am Soc Nephrol 9: 1080-1084, 1998.

39. Buyan N, Bideci A, Ozkaya O, et al: Leptin and resistin levels and their relationships with glucose metabolism in children with chronic renal insufficiency and undergoing dialysis. Nephrology (Carlton) 11: 192-196, 2006.

40. Yang FF, Lin X, Wang J and Luo ZW: Effect observation of peritoneal dialysis on clearance of hyperleptinemia and inflammatory factor in uremic patients. Chongqing Yi Yao 40: 34-35,37 2011 (In Chinese)

41. Xu XD, Hu P, Wang NS, et al: The relationship between leptin and nutrition status in patients with chronic renal failure undergoing different ways of blood purification. Xuzhou Yi Ke Da Xue 28: 85-88, 2008 (In Chinese)

42. Wu R, Ma K and Wang J: Analysis of serum leptin and inflammatory cytokines in peritoneal dialysis clear uremic patients. Chin and Foreign Health Abstract 8: 468-468, 2011.

43. Wu JQ, Zhou ZS, Pan ZX and Li HZ: An analysis for the relationship between leptin and the nutrition status in peritoneal dialysis patients. Zhongguo Ji Ceng Yi Yao 13: 185-186, 2006 (In Chinese)

44. Wang ZM, Wang Q and Yu JS: Effects of peritoneal dialysis on indexes in patients with chronic renal failure. Xian Dai Zhong Xi Yi Jie He Za Zhi 19: 1185-1186, 1226, 2010 (In Chinese)

45. Zuo J, Zou JZ, Fang Y, et al: Serum leptin levels and malnutrition in chronic renal failure. Shanghai Yi Xue 679-682, 2002 (In Chinese).

46. Ma Y, Zhao J, Sun XW, et al: Serum leptin levels and its clinical significance in patients with different stages of chronic renal failure. Zhong Guo Xian Dai Yi Yao Za Zhi 13: 42-45, 2011 (In Chinese).

47. Guan X and Zheng HG: Analysis of leptin levels in peritoneal dialysis patients and its influencing factors. Zhong Guo Shi Yong Nei Ke Za Zhi 26: 19-21, 2006 (In Chinese). 
48. An WS, Son YK, Kim SE, et al: Association of adiponectin and leptin with serum lipids and erythrocyte omega-3 and omega-6 fatty acids in dialysis patients. Clin Nephrol 75: 195-203, 2011.

49. Hou N and Luo JD: Leptin and cardiovascular diseases. Clin Exp Pharmacol Physiol 38: 905-913, 2011.

50. Díez JJ, Bossola M, Fernández-Reyes MJ, et al: Relationship between leptin and all-cause and cardiovascular mortality in chronic hemodialysis patients. Nefrologia 31: 206-212, 2011.

51. Golembiewska E, Safranow K, Ciechanowski K, et al: Adipokines and parameters of peritoneal membrane transport in newly started peritoneal dialysis patients. Acta Biochim Pol 60: 617-621, 2013 .
52. Beberashvili I, Sinuani I, Azar A, et al: Longitudinal study of leptin levels in chronic hemodialysis patients. Nutr J 10: 68, 2011.

53. Ponce D, Caramori JT, Barretti P and Balbi AL: Peritoneal dialysis in acute kidney injury: Brazilian experience. Perit Dial Int 32: 242-246, 2012.

54. Fontán MP, Rodríguez-Carmona A, Cordido F and García-Buela J: Hyperleptinemia in uremic patients undergoing conservative management, peritoneal dialysis and hemodialysis: A comparative analysis. Am J Kidney Dis 34: 824-831, 1999.

55. Tsai JP, Tsai CC, Liu HM, et al: Hyperleptinaemia positively correlated with metabolic syndrome in hemodialysis patients. Eur J Intern Med 22: e105-e109, 2011. 